\title{
AFFECTIVE SOCIETTIES
}

Margreth Lünenborg

Soziale Medien, Emotionen und Affekte

SFB 1171 Working Paper 01 / 20

Berlin 2016 - ISSN 2509-3827 


\section{SFB Affective Societies - Working Papers}

Die Working Papers werden herausgegeben von dem an der Freien Universität Berlin angesiedelten Sonderforschungsbereich 1171 Affective Societies - Dynamiken des Zusammenlebens in bewegten Welten und sind auf der Website des SFB sowie dem Dokumentenserver der Freien Universität Berlin kostenfrei abrufbar:

www.sfb-affective-societies.de und http://edocs.fu-berlin.de

Die Veröffentlichung erfolgt nach Begutachtung durch den SFB-Vorstand. Mit Zusendung des Typoskripts überträgt die Autorin/der Autor dem Sonderforschungsbereich ein nichtexklusives Nutzungsrecht zur dauerhaften Hinterlegung des Dokuments auf der Website des SFB 1171 sowie dem Dokumentenserver der Freien Universität. Die Wahrung von Sperrfristen sowie von Urheber- und Verwertungsrechten Dritter obliegt den Autorinnen und Autoren. Die Veröffentlichung eines Beitrages als Preprint in den Working Papers ist kein Ausschlussgrund für eine anschließende Publikation in einem anderen Format. Das Urheberrecht verbleibt grundsätzlich bei den Autor/innen.

Zitationsangabe für diesen Beitrag

Lünenborg, M. (2020). Soziale Medien, Emotionen und Affekte. Working Paper SFB 1171 Affective Societies $01 / 20$.

Static URL: https://refubium.fu-berlin.de/handle/fub188/17614

Working Paper ISSN 2509-3827

Diese Publikation wurde gefördert von der Deutschen Forschungsgemeinschaft (DFG).

Sonderforschungsbereich 1171

Affective Societies

Freie Universität Berlin

Habelschwerdter Allee 45

14195 Berlin

E-Mail: office@sfb1171.de 


\title{
Soziale Medien, Emotionen und Affekte
}

\author{
Margreth Lünenborg
}

30.07 .2020

\begin{abstract}
Der Beitrag liefert einen Überblick über das Verhältnis von sozialen Medien und Emotionen sowie Affekten. Nach einer Einführung in die sozialwissenschaftliche Emotionsforschung und affect studies, die Emotionen als wesentliche Bausteine sozialer Ordnung sichtbar machen, werden die wechselseitig konstituierenden Bedingungen von Emotionen und sozialen Medien herausgearbeitet. Emotionen werden medial erzeugt, geteilt, archiviert und reguliert. Soziale Medien lassen sich damit als Affektgeneratoren fassen. Die Architektur sozialer Netzwerkplattformen zielt maßgeblich auf die Affizierung ihrer Nutzer*innen ab. Durch vielfach geteilten Hass oder Empörung, Erregung oder empathische Solidarität wird die Verweildauer auf der Plattform und die Intensität der Nutzung gesteigert. Solche Interaktionen lassen sich als affektive Medienpraktiken fassen. Sie können bspw. in Form von Memes als kritische, ironische oder subversive Aneignung von Medienangeboten auftreten, die jedoch den ökonomisch grundierten Affordanzen der Plattform nicht entkommen können. Auf diese Weise werden sie zu wesentlichen Bestandteilen einer affektiven Ökonomie.
\end{abstract}

\section{Einleitung}

Beispiele für Emotionen und Affekte in den sozialen Medien lassen sich zahlreich finden: So kommen Hass, Aggression oder Wut in Online-Kommentaren zum Ausdruck und fordern Social-Media-Teams heraus, einen angemessenen Umgang mit diesen ÄuBerungen zu finden. Bei der kreativen und oftmals subversiven Aneignung von Bildern und Videos durch User entstehen Memes, die von den Betrachtenden als ironisch oder satirisch, mit Häme oder Freude gelesen und weiterverbreitet werden. Öffentlich sichtbare und ausgestellte Gefühle von Intimität und Nähe können, wenn sie als unangemessen wahrgenommen werden, bei unbeteiligten Beobachter*innen Fremdscham erzeugen. Begeisterung und Zustimmung drückt sich im vielfachen Teilen von Informationen über soziale Medien aus. So entstehen Wellen von Solidarität, Mitgefühl und Empathie in digitalen Netzwerken und überschreiten dabei nationale Grenzen sowie kulturelle und soziale Differenzen. Mit Bezeichnungen wie ,Shitstorm‘, ,Candystorm ‘ oder ,Empörungskaskaden“ haben sich Begriffe etabliert, die die Dynamik von Emotionen in sozialen Medien beschreiben wollen. Gibt es also spezifische Formen, in digitalen Netzwerken zu lieben und zu hassen, zu trauern und sich zu begeistern?

„Twitter ist, wie kein anderes digitales Medium so aggressiv und in keinem anderen Medium gibt es so viel Hass, Böswilligkeit und Hetze. Offenbar triggert Twitter in mir etwas an: aggressiver, lauter, polemischer und zugespitzter zu sein“ (Habeck 2019). Mit dieser Begründung entscheidet sich der Grünen-Vorsitzende Habeck im Januar 
2019, seinen Twitter Account zu löschen. Er suggeriert damit, die Architektur einer digitalen Plattform generiere, forme und strukturiere Emotionen in einer eigenständigen Weise. Danach stellt sich die Frage, wie sich also das Verhältnis von Emotionen, Affekten und digitalen Kommunikationsstrukturen beschreiben lässt. Welche Bedeutung haben solche dynamischen Artikulationen von Emotionen für die Gesellschaft?

Um diese Fragen zu beantworten, ist es im ersten Schritt erforderlich, grundlegender die Bedeutung von Emotionen und Affekten für die Herstellung sozialer Ordnung zu verstehen. Dazu liefert dieser Beitrag Grundlagen aus der Emotionssoziologie sowie der Affektforschung. Im zweiten Schritt diskutiert er, wie ein solches Verständnis von Emotionen auf Medienpraktiken in sozialen Medien übertragbar ist. Welche affektiven Dynamiken zwischen Usern und dem Medium werden hier wirksam? Es wird Einsicht in aktuelle Forschung zum Verhältnis von Emotionen und sozialen Medien gegeben.

Dabei stehen einerseits Empörung und ,hate speech`als Modi der Kommunikation im Fokus, die normative Grundlagen von Öffentlichkeiten bedrohen. Andererseits werden mit Formen der transnationalen Empathie und Solidarität affektive Modi der Vergemeinschaftung in den Blick genommen. Schließlich wird mit den Konzepten ,affective news stream' (Papacharissi 2014; Papacharissi und de Fatima Oliveira 2012) und ,affective publics' (Lünenborg 2019; Papacharissi 2015) erörtert, in welcher Weise die Zirkulation von Affekten und Emotionen in sozialen Medien die Formierung von Öffentlichkeiten beeinflusst und prägt. So wird sichtbar, dass keineswegs erst mit der Verfügbarkeit von digitalen Netzwerken Emotionen auf die Formierung von Öffentlichkeiten Einfluss genommen haben. Vielmehr hat die Forschung unter den Bedingungen digitaler Kommunikation die Bedeutung von Emotionen für öffentliche Kommunikation verstärkt in den Blick genommen und damit die vormals präferierten Konzepte primär rationaler Diskurse (Habermas 1962) modifiziert und erweitert. Auf dieser Grundlage wird das wechselseitig konstituierende Verhältnis von Emotionen und sozialen Medien aufgezeigt.

\section{Emotionen und Affekte als Elemente sozialer Ordnung}

Was genau sind Emotionen und Affekte? Darüber herrscht in der Wissenschaft keineswegs Einigkeit, vielmehr existieren vielfältige, teils gegensätzliche Vorstellungen davon, was genau Emotionen ausmacht, wovon sie beeinflusst werden und welche Einflüsse sie selbst wiederum auf die Gesellschaft haben. Entsprechend sind auch die Vorstellungen davon, in welchem Verhältnis Emotionen, Affekte und gesellschaftliche 
Ordnung zueinander stehen, durchaus unterschiedlich. Während ein großer Teil der psychologischen und neurowissenschaftlichen Forschung davon ausgeht, dass Emotionen angeboren, stabil, klar definiert und universal sind (Ekman 1992; Scherer 2005), verfügt die sozialwissenschaftliche Forschung über eine langjährige Tradition, in der Emotionen als durch und durch sozial und kulturell geformt begriffen werden. Wem gegenüber wir Respekt empfinden, wer sich beschämt fühlt oder in welcher Weise Schuld erlebt wird, das hängt in hohem Maße mit gesellschaftlichen Machtpositionen, Hierarchie und Ungleichheiten zusammen. Zugleich können Emotionen selbst soziale Strukturen schaffen: So ist die Liebe zwischen Eltern und Kind konstitutiv für eben dieses soziale Verhältnis. Auch wenn Emotionen individuell zugerechnet werden (jemand ist wütend oder glücklich), so sind sie doch als soziale und kulturelle Struktur zu betrachten, um ihre Bedeutung für die Konstituierung von sozialer Ordnung (oder für deren Brüchigkeit) angemessen erfassen zu können.

Arbeiten zu Scham (Neckel 1991) oder Demütigung (Frevert 2017) machen sichtbar, in welcher Weise mittels Emotionen auch in historischer Perspektive soziale Ordnung geschaffen, aufrecht erhalten oder irritiert wird. Illouz $(2003 ; 2016 ; 2018)$ hat eindrucksvoll gezeigt, wie Liebe als Gefühl unter kapitalistischen Bedingungen zu einem warenförmigen Gut geworden ist. Hochschilds (1979) frühe Konzepte von emotion work und feeling rules machen zudem deutlich, dass das Empfinden und Zeigen spezifischer Emotionen ein zutiefst sozialer Lernprozess ist, der in professionellen Beziehungen ebenso stattfindet wie in privaten. Als Bausteine sozialer Ordnung organisieren solche Gefühle Gemeinschaften, also ein ,Wir ${ }^{\star}$ und ein ,Ihr'. So konstatieren von Scheve und Berg (2018, S. 27), die vorhandene Emotionsforschung habe für die „doppelte Bedeutung von Emotionen hinsichtlich des Verständnisses von Gemeinschaft und Gesellschaft sensibilisiert: Einerseits stellen Emotionen soziale Bindungen und Kohäsion her, andererseits sind sie unübersehbare Triebfedern von Konflikt und Desintegration. “ In diesen Arbeiten werden Emotionen zumeist als diskrete, episodische, explizite und kulturell elaborierte Phänomene verstanden. In der Verschränkung von körperlichen Reaktionen, Bewertungen und innerem Erleben treten sie für begrenzte zeitliche Dauer auf. Scham, Freude, Hass oder Schuld empfinden wir in der Regel in einer konkreten Situation mit einer spezifischen Intensität und Gerichtetheit.

Ergänzend steht mit dem Begriff des Affekts ein Konzept zur Verfügung, das stärker latente, dauerhafte, fluide Intensitäten zwischen Körpern - humanen wie non-humanen - in den Blick rückt. Mit einem solchen weiten Verständnis von Körpern werden auch Artefakte und Technologien eingeschlossen, also mit Screens oder wearable technologies eine Vielzahl von Objekten, die für digitale Kommunikation eine zentrale 
Rolle spielen. Mit dem turn to affect (Clough und Halley 2007; Lünenborg und Maier 2018) in kultur- wie sozialwissenschaftlicher Forschung hat sich auch die Analyse von Medien und deren Nutzung auf Fragestellungen jenseits des Repräsentationalen erweitert. Damit ist gemeint, dass Medien nicht allein auf der Ebene von Aussagen und Diskursen Bedeutung erzeugen, sondern auch unmittelbar - eben affektiv - Spannung und Erregung, Unlust oder Beruhigung vermitteln. Bei einer Vielzahl von Affekt-Begriffen, die in den Kultur- und Sozialwissenschaften verwandt werden (dazu als Auswahl und Überblick: Blackman und Venn 2010; Gregg und Seigworth 2010; Penz und Sauer 2016; Slaby 2018; Wetherell 2012), soll der Affekt hier charakterisiert sein als jenes evaluative Empfinden, das intensiv an körperliche Erfahrungen anknüpft und die Form des In-die Welt-gestellt-Seins beschreibt.

In dieser Weise lassen sich Affekte als Rohmaterial von Emotionen betrachten; sie sind nicht propositional, (noch) nicht geformt, dynamisch und relational, also fortwährend reaktiv mit Blick auf andere Köper und Umgebungen. In dieser Weise ist es ein zentrales Charakteristikum von Körpern - insbesondere von menschlichen Körpern zu affizieren und affiziert zu werden, also selbst Affekte auszudrücken und in der Lage zu sein, bei anderen (menschlichen) Körpern Affekte zu erzeugen. So formen und gestalten menschliche Körper ihren Weltbezug. Mit diesem Verständnis von Affekt sollen jene Stimmungen, Atmosphären und Empfindungen gefasst sein, die beständig unser Sein begleiten, also ubiquitär sind, gleichwohl wechselnd in Intensität und Valenz auftreten und (mitunter) wahrgenommen werden.

Affekte werden hier mit Wetherell (2012) nicht als Gegensatz zu Diskursen gefasst, sondern als mit diesen verschränkt. Auch wenn Affekte nicht unmittelbar als Signifikant erscheinen - also nicht direkt als Zeichen für eine eindeutige Bedeutung gelesen werden können - und damit nicht unmittelbar auf eine repräsentationale Ebene verweisen, so treten sie doch bedeutungsstiftend auf. Im Anschluss an Wetherell (2012) und Reckwitz (2017) lassen sich Affekte als grundierendes Element von sozialen Praktiken begreifen. Als affective practice lässt sich damit jenes zumeist ritualisiert und unbewusst verlaufende Handeln beschreiben, ,[which] rests on a large unarticulated hinterland of possible semiotic connections and meaning trajectories (built around the discursive, the visual, the tactile etc.)" (Wetherell 2012, S. 129). Reckwitz (2017, S. 116) betont dabei die Relevanz von Affekten für die Entstehung sozialer Strukturen: „There can consequently be no social order without affect, but there can be vastly different types and intensities of affects within practices." In diesem Sinne lassen sich Affekte als evaluative Gefühle verstehen - Lust oder Unlust, Anspannung, Gereiztheit oder Ruhe -, die Menschen kontinuierlich begleiten, dabei in Intensität, 
Valenz und Modus beträchtlich schwanken. Die kultur- und sozialwissenschaftlichen affect studies haben sich mit diesem Konzept kritisch aktuellen Formen der Organisation von Arbeit (Gregg 2011; Penz und Sauer 2016), der Formierung von Körper und Geschlecht (Blackman 2012; Blackman und Venn 2010; Sauer und Penz 2014) oder der gesellschaftlichen Sedimentierung von Rassismus (Ahmed 2004a; 2004b) zugewandt.

Während Emotionen also als kulturell geformte und elaborierte Konzepte sozialer Relationalität verstanden werden, fasst der Begriff des Affekts jene latenten Kräfte und Dynamiken zwischen Menschen, Artefakten und Technologien, die das Rohmaterial von Emotionen bilden, menschliches Handeln motivieren, jedoch noch nicht eindeutig kodiert sind. Dabei wird die Unterscheidung zwischen Emotionen und Affekten nicht trennscharf vorgenommen. Für beides - Emotionen wie Affekte - gilt, dass sie wesentliche Bausteine sozialer Ordnung sind und Prozessen der Kommunikation stets innewohnen. Damit erhalten sie eine zentrale Bedeutung für das Verständnis medialer Kommunikationsprozesse und insbesondere der Kommunikation in sozialen Medien.

\section{Emotionen und Affekte in sozialen Medien}

Im Folgenden wird knapp skizziert, in welcher Weise Medien mit der gesellschaftlichen Erzeugung, Verbreitung und In-Wert-Setzung von Emotionen und Affekten verbunden sind. Medien lassen sich als maßgebliche Orte, Strukturen und Institutionen der Inszenierung, Herstellung und Zirkulation von Emotionen und Affekten verstehen. Auf der Ebene der Medieninhalte lassen sich Emotionen als impliziter Gegenstand sowie als Muster narrativer Strukturen erkennen. Mediale Erzählungen liefern feeling rules, indem sie vermitteln, welche Emotionen als angemessen gelten und wie diese performiert werden sollen. Die Erzeugung von Medienprodukten ist mit Emotionsarbeit auf Seiten der Produzent*innen verbunden - und sei es durch die spezifischen Anstrengungen, die unternommen werden, um beispielsweise die journalistische Nachricht frei von Emotionen erscheinen zu lassen (Wahl-Jorgensen 2018), oder wie Anderson es formuliert ,the avoidance of passion in factual analysis is itself a passionate endeavor" (Anderson 2018, S. 4). Auf Seiten der Rezeption spielen Emotionen eine wesentliche Rolle bei der Auswahl von, Zuwendung zu und Verarbeitung von Medienangeboten (Konijn 2013).

Wahl-Jorgensen (2019, S. 49) beschreibt medienvermittelte Emotionen (mediated emotions) - insbesondere in der politischen Berichterstattung - als performativ, diskursiv hergestellt sowie kollektiv bedeutsam und politisch. Sie räumt damit Emotionen 
einen wichtigen Raum im politischen Diskurs ein. Mit der Zirkulation in der Öffentlichkeit lösen sie sich aus der Verortung innerhalb einzelner Körper. In der journalistischen Berichterstattung findet die performative Herstellung von Emotionen in der Regel in doppelter Hinsicht statt: Akteur*innen im politischen Feld zeigen bzw. erzeugen Emotionen, die dann wiederum vom Journalismus in spezifischen Darstellungsund Erzählweisen als Emotionen zum Ausdruck gebracht werden. Weil zudem die beteiligten Kommunikator*innen strategische Kommunikationsabsichten verfolgen, ist der Authentizitätsgehalt der medial sichtbaren Emotionen schwer oder gar nicht einzuschätzen. Zugleich ist unübersehbar, dass medienvermittelte Emotionen stets symbolisch und diskursiv hergestellt sind. Mit Sprache, Bild und Ton - ggf. multimodal werden Emotionen unter Rückgriff auf Erzählkonventionen und Präsentationsstile gezielt erzeugt. In historischer Perspektive ist dabei deutlich erkennbar, wie sich z.B. Konventionen zum Herstellen von Stolz, Schuld oder Scham in Medien fortlaufend wandeln.

Im Unterschied zu einer solchen doppelten Zuschreibung von Emotionen in nichtfiktionaler Berichterstattung erfolgt sie in sozialen Medien ungleich komplexer und widersprüchlicher. Konventionen der Regulation von Emotionen, die etablierten Mediengattungen eingeschrieben sind, werden hier außer Kraft gesetzt. Stattdessen ermöglichen digitale Plattformen den spontanen, unmittelbaren Gefühlsausdruck von Freude und Begeisterung ebenso wie die strategisch entwickelte Artikulation von Stolz, die Neid, Beschämung oder Unbehagen anderer Nutzer*innen erzeugt oder gar präzis auf diese abzielt. Die Entgrenzung zwischen Individual- und Massenkommunikation, die für soziale Medien charakteristisch ist, erzeugt emotionstheoretisch Kontingenz, Widersprüchlichkeit und Unsicherheit.

Intime Nähe und komplexe Öffentlichkeit lassen sich in sozialen Medien nicht kategorial unterscheiden, sondern treten unmittelbar nebeneinander auf. Persönliche (Gefühls-) Äußerungen können in professionellen Kontexten als unangemessen bewertet werden. Nikunen nutzt vor diesem Hintergrund Hochschilds Begriff der Emotionsarbeit, um zu verdeutlichen, welch komplexes Navigieren zwischen Gefühlen erforderlich ist: "As the spheres of personal and professional become increasingly intertwined in the digital era, the demands of emotional labour are amplified" (Nikunen 2019, S. 332).

Dabei bedürfen medial erzeugte Emotionen (mediated emotions) in sozialen Medien keiner außermedialen Emotionsäußerungen, auf die Bezug genommen wird. Vielmehr lässt sich das Handeln mit und in sozialen Medien selbst als zutiefst affektive Praxis begreifen. Mit dem Konzept der affektiven Medienpraktiken (affective media 
practices; Lünenborg und Maier 2019; Nikunen 2018) lassen sich also im ersten Schritt Formen des Teilens, Likens und Kommentierens beschreiben. So irritieren affektive Medienpraktiken von Usern jene tradierten Selektions- und Distributionslogiken, die den Journalismus historisch mit der Autorität zur Herstellung und Bereitstellung relevanter Themen zur öffentlichen Kommunikation versehen haben. Weitergehend umfassen affektive Medienpraktiken jene affektiv geladene Zuwendung zu und Involviertheit in Kommunikationsprozesse mit sozialen Medien. Relevant wird hier also nicht das Verhältnis von außermedial vorhandenen Emotionen und deren Repräsentation in Medien(texten), sondern der affektive Gehalt des Agierens mit Medien selbst. In diesem Sinne bezeichnet van Dijcks Analyse des ,engineering sociality in a culture of connectivity“ (2013, S. 3) einen zutiefst affektiven Prozess: In Verbindung sein, Verbindungen herstellen sowie aus relevanten Verbindungen ausschließen oder selbst ausgeschlossen zu sein - also das Herstellen und Aufrechterhalten von Sozialität mittels digitaler Plattformen - basiert auf fortlaufender affektiver Arbeit aller Beteiligten.

Analytisch von Interesse sind dabei einerseits die spezifischen technologischen Affordanzen, mit denen Plattformen die Erzeugung von Affekten präfigurieren (z. B. der Like-Button), die symbolischen Ausdrucksformen von Emotionen, die sich in sozialen Medien etabliert haben und von dort aus Eingang in andere private und öffentliche Kommunikationsformen genommen haben (z. B. Emoticons oder Memes). Bedeutsam ist auch die Kontrolle und Regulation von Emotionen, die von Usern in Interaktion ebenso geleistet wird wie von Community Manager*innen, Plattformbetreiber*innen oder der Rechtsprechung. Wie eng dabei die Verbindung zwischen Emotionen und Medien gestaltet ist, zeigt sich an neueren Formen von sensitive media, also sensorgestützten digitalen Geräten, die unmittelbar auf Puls- oder Atemfrequenz, Herzschlag oder Hautspannung reagieren. Indem die dort generierten Daten in sozialen Medien geteilt werden, sind diesen Formen der affektiven Vermessung und Optimierung des eigenen Körpers unmittelbar Charakteristika des Vergleichens und Wettbewerbs eingeschrieben. Malinowska und Miller beschreiben diese materialisierten Formen der Interaktion zwischen menschlichem Körper und digitaler Technologie als "emotionally capable in how they convey, reflect, and respond to human needs" (Malinowska und Miller 2017, S. 661). Emoticons in der Schriftsprache oder sprachliche Interaktion mit Sprachassistenten imitieren menschliche Emotionsäußerungen und entwickeln diese dabei zugleich weiter - wenn bspw. das Zwinkersmiley Eingang in die Alltagskommunikation findet und in seiner kontingenten Unschärfe jederzeit ironische Distanzierung vom Gesagten ermöglicht. Personalisierte User-Interfaces, individuelle 
Klingeltöne, das persönliche Google-Geburtstagslogo oder die persönliche FacebookSlideshow, all das trägt zur emotionalen Bindung von Nutzer*innen an soziale Medien bei: „Seemingly practical, they bind us emotionally. And we seek in them emotional succour and a public imaging of our private identities" (Malinowska und Miller 2017, S. 662).

Diese emotionale Bindung lässt sich als stetige Interaktionsschleife zwischen Nutzer*in und Medium beschreiben. Boler und Davis schlagen dafür den Begriff des affective feedback loop vor, ,[to] describe the emotional and affective circuit of relationality between human and information in computer-mediated environments" (2018, S. 83). Sie begreifen diesen Kreislauf als grundsätzlich unabgeschlossene Wiederholung von affektiver Äußerung und Reaktion - in Form einer positiven Verstärkung als Belohnung oder als negative Reaktion und Unzufriedenheit, die dann wiederum neue affektive Impulse erzeugt. Sie entwerfen ihr Konzept vor dem Hintergrund der polarisierten politischen Öffentlichkeit in den USA nach dem Amtsantritt von Donald Trump. Doch die beschriebene Dynamik lässt sich auf andere Diskurse übertragen: Die algorithmisch formierte Struktur der Plattform zielt auf möglichst lange Verweildauer und entsprechend zielgenaue affektive Adressierung der Nutzer*innen ab:

,Without the algorithmic reinforcing of feedback (or, ,rewards"), modulated to induce the affective state of , user-satisfaction ' - which may be composed of differing emotions, such as outrage or pleasure - users would quickly cease engaging commercial platforms such as Facebook" (Boler und Davis 2018, S. 83).

So wird deutlich, dass die ökonomisch getriebene Architektur digitaler Plattformen auf eine stete Affektlenkung sowie Steuerung der Intensität und Dauer von Affekten abzielt.

Vor diesem Hintergrund erscheint die insbesondere in der Kommunikationswissenschaft dominierende, vorrangige Fokussierung auf (erwünschte) Deliberation in Online-Diskursen verkürzt, verliert sie doch diese basal treibende Dynamik der Affektlenkung und -steuerung durch die Architektur der Plattformen aus dem Blick. Hier zeigt sich nicht Deliberation, sondern affective attunement, also die affektive Bindung von Nutzer*innen, als primäres Ziel von Kommunikation in digitalen Netzwerkplattformen. Dieses Spannungsverhältnis im Blick behaltend werden im nächsten Schritt spezifische Muster von Emotionsäußerungen und Affektdynamiken in sozialen Medien dargestellt und ihre Bedeutung für öffentliche Kommunikation diskutiert.

\section{Empörungskaskaden und ,hate speech'als affektive Dynamiken}


Als Empörungskaskaden oder -wellen lassen sich jene Kommunikationsprozesse in sozialen Medien bezeichnen, bei denen User ,über die dialogisch aufgebauten Kommunikationskanäle von Unternehmen, Organisationen oder Privatpersonen diese öffentlich kritisieren und dabei oft aggressiv und sogar beleidigend auftreten. Zusätzlich handelt es sich bei einem Shitstorm immer um eine, wenn auch subjektiv betrachtete, große Anzahl dieser negativen Meinungsäußerungen, die sich in manchen Fällen nicht mehr auf das ursprüngliche Thema der Kritik beziehen, sondern unabhängig vom eigentlichen Auslöser lediglich eine Diffamierung als Ziel verfolgen“" (Spiller und Hintzen 2016, S. 110). Mit dem Begriff des ,Shitstorms` (online fire storm) hat diese affektive Kommunikationsdynamik eine Bezeichnung gefunden, die auch alltagssprachlich breit genutzt wird. Entgegen naiv emotionstheoretischer Annahmen weist Haarkötter darauf hin, ,dass die prägenden Kommentare eines Shitstorms nicht so sehr emotional-eruptiv und unbewusst, sondern im Gegenteil eher rational-bewusst im Sinne einer rhetorischen Kommunikationsstrategie vorgebracht werden“" (2016, S. 45). Mit Pfeffer et al. (2014) lässt sich die Charakteristik dieser Kommunikationsfiguration beschreiben entlang ihrer zeitlichen Geschwindigkeit (speed and volume), der Polarisierung (binary choices), die mit dem gänzlichen Fehlen diskursiver Auseinandersetzung einhergeht, der Verbreitung entlang lokaler oder translokaler Netzwerke (network clusters), die zudem auf hoher sozialer Homogenität basieren (lack of diversity). Durch crossmediale Verbreitung erhöht sich die Reichweite solcher Empörungsdynamiken zudem, wenn ein Spill Over von sozialen Medien in klassisch journalistische Medien stattfindet.

Das performative Hervorbringen kollektiver Emotionsäußerungen als strategisches Mittel der Kommunikation wird hier als Relation zwischen technischen Affordanzen und User-Aktivitäten sichtbar. Die massenhaften beleidigenden oder aggressiven Äußerungen erzeugen in der Dynamik ihrer Verbreitung eine Form von Öffentlichkeit, die für Einzelpersonen oder auch Organisationen öffentliche Beschämung zur Folge hat, die der des historischen Prangers ähnlich sein können (vgl. Frevert 2017). Die Historikerin Frevert spricht bei der „Beschämung online“ davon, dass sich hier Beschämung von ihrem gesellschaftlichen Rahmen und ihrer eigentlichen Funktionen gelöst habe. Habe Scham und Beschämung gesellschaftlich grundlegend den Sinn, Normverstöße zu ahnden und damit die Integration sozialer Gruppen und Gemeinschaften sicherzustellen, so sei diese Funktion in digitalen Netzwerken verloren gegangen: „Im Zentrum steht vielmehr die Demütigung als Demütigung, der Spaß an Erniedrigung, Beschämung als Selbstzweck“ (Frevert 2017, S. 141). Ihr Argument: 
Durch soziale ,Entbettung“ in digitalen Netzwerken verliert die gesellschaftliche Regulation von Emotionen an Bedeutung. Demütigung dient dann allein dem Zweck der Exklusion.

Vor diesem Hintergrund befasst sich medien- und kommunikationswissenschaftliche, aber auch juristische und informationstechnologische Forschung intensiv mit den Dynamiken emotionsgetriebener öffentlicher Kommunikation in sozialen Medien und deren negativen Folgen für die Öffentlichkeit, zumeist unter dem Begriff hate speech zusammengefasst (Banks 2010; Erjavec und Kovačič 2012; Gagliardone 2019; Wagner 2019). Dies erscheint umso drängender, je mehr Aggressivität, Hass oder Beleidigungen die Kommunikation in sozialen Medien kennzeichnen. Das Gros der Forschung geht dabei vom Ideal deliberativer Kommunikation aus, bei der in sachlichem, neutralem und respektvollem Modus Argumente gleichberechtigt ausgetauscht werden sollten. An das Habermas'sche Ideal des rationalen Diskurses angelehnt, sollte demnach Kommunikation möglichst frei von Emotionen erfolgen. Erscheint diese normative Annahme vor dem eingangs diskutierten Verständnis einer affektiven Grundierung jeglicher sozialen Praxis problematisch (vgl. dazu weitergehend den Abschnitt Affective Publics unten), so bleibt das Bemühen um konstruktive Moderation von Debatten und Vermeidung bzw. Ausschluss beleidigender, rassistischer oder sexistischer Äußerungen gleichwohl nachvollziehbar und wünschenswert. Neben einschlägiger Forschung hat dies auch konkrete Initiativen von Nutzer*innen (\#IchBinHier; Ley 2018) wie von Medienakteur*innen, etwa den Neuen Deutschen Medienmacher*innen (2018) und ihrem Projekt NoHateSpeech, ausgelöst.

Kramp und Weichert (2018, S. 9) rücken in ihrer praxisorientierten Analyse „bewusste Grenzverletzungen in Nutzerdiskursen“ in den Fokus und begreifen „deren Regulierung [als] eine medienpädagogische Schlüsselaufgabe von und für Journalistinnen und Journalisten des 21. Jahrhunderts“. Mit einer konkreten Handreichung, die die journalistische Autorität in Diskussionsforen stärken will, empfehlen sie Diskursinterventionen, Verzicht auf Ironie und Zynismus sowie, wo erforderlich, die strafrechtliche Verfolgung von Hasskriminalität.

Unter der zusammenfassenden Bezeichnung ,uncivil behaviour“ untersuchen eine Vielzahl von Studien empirisch die Interventionsmöglichkeiten von Community-Manager*innen oder Social-Media-Redaktionen in Reaktion auf Hasskommentare (vgl. als Überblick Ziegele u. a. (2019); grundlegend Ziegele \& Jost (2016), Ziegele, Koehler \& Weber (2018), Naab et al. (2018)). Sie kommen auf der Basis von Inhaltsanalysen zu dem Ergebnis, dass die diskursive Qualität in Online-Foren erhöht werden kann, wenn Community-Manager*innen ,einen respektvollen, vergemeinschaftenden Ton 
treffen und sachlich mitdiskutieren“ (Ziegele u. a. 2019, S. 7). Dies erhöht die Partizipationsbereitschaft der User. Voraussetzung dafür sei, dass „die Kommentare selbst deliberative Kriterien wie Respekt und Diskursivität erfüllen“ (Ziegele u. a. 2019, S. $8)$.

Emotionstheoretisch muss der Rekurs auf den ,unzivilen' Charakter von OnlineDebatten unbefriedigend bleiben, ersetzt hier doch eine Bewertung des Effekts die eigentliche Beschreibung des emotionalen Ausdrucks. Es liegt damit nahe, dass auf diese Weise die (negative) Bewertung von Menschen oder Menschengruppen als normverletzend gefasst wird, anstelle des eigentlichen Emotionsausdrucks und seines affizierenden Gehalts für andere Nutzer*innen. Darauf verweisen insbesondere auch vielfältige englischsprachige Untersuchungen, die den Begriff harmful speech sowie in Einzelfällen online harassment wählen (Faris u. a. 2016). So zeigen auch Sellars' (2016) umfängliche Definitionsbemühungen, dass hier ganz vorrangig auf intendierte Verletzungen, Herabwürdigungen und Erniedrigungen von Menschen oder Menschengruppen abgezielt wird, mithin auf den Effekt von Hass, jedoch bislang kaum Ansätze zur Beschreibung und Erfassung der Emotion und der durch sie erzeugten Affizierung der User vorliegen. Entsprechend setzen die Vorschläge zur Regulation von Online-Diskursen bei der Vermeidung von Effekten des Ausschlusses und der Diskriminierung an, greifen also auf rationale Steuerungspraktiken zur Verhinderung als unerwünscht beschriebener Effekte zurück.

Was jedoch könnte hier eine emotionstheoretische Betrachtung beitragen? Ahmed (2004a, 2004b) bietet mit ihrem Konzept der „Affective Economies“ einen solchen Fokus (vgl. auch Lehmann et al. 2019). Grundlegend stellt sie dar, wie Emotionen in ihrer gesellschaftlichen Zirkulation als Tauschökonomie wirksam werden. „Emotions work as a form of capital: affect does not reside positively in the sign or commodity, but is produced only as an effect of its circulation" (Ahmed 2004a, S. 118). Die Wirkmächtigkeit dieser Ökonomie macht Ahmed beispielhaft in ihrer Analyse der OnlineSelbstbeschreibung der Aryans Nation sichtbar; hier wird Liebe und Hass unverbrüchlich an Weiße und Schwarze gebunden. Rassismus wird erzeugt, indem Hass auf Schwarze als ,emotionales Bindemittel' einer imaginierten Weißen Nation fungiert. Ahmed macht hier deutlich, wie Emotionen und Affekte relational als Kräfte wirksam werden, die Verbindungen zwischen dem Individuum und einer sozialen Struktur, hier der Nation, herzustellen in der Lage sind. Sie zeigt wie Emotionen gebunden an Zeichen, Bilder oder Texte wirkmächtig zirkulieren und als ,affective politics of fear" (Ahmed 2004b, S. 62-81) insbesondere rechtspopulistische und rechtsautoritäre Akteur*innen von ihnen Gebrauch machen. Koppetsch knüpft an diese Diskurse an 
und beschreibt Formen der „Zornbewirtschaftung“ (Koppetsch 2019, S. 229) als affektive Modulation rechtspopulistischer Diskurse, in denen „Zornkollektive[...] und gekränkte[..] ,Zivilisationen““(S. 232) sich artikulieren.

\section{Solidarität und Empathie: Aktivismus und Memes als affektive Praktiken}

Sind die Analysen von hate speech und Empörungskaskaden in sozialen Medien primär getrieben von der Sorge um die destruktiven, anti-sozialen Kräfte, die den erwünschten Formen der Deliberation zuwider laufen, erweist sich ein Blick auf emotionale und affektive Potenziale sozialer Medien als nicht minder ertragreich. So lassen sich eine Vielzahl an Protestformen (Occupy Wallstreet \#OWS; \#BlackLivesMatter; \#FridaysForFuture) oder Solidarisierungskampagnen (\#RhodeMustFall) benennen, in denen mit und durch soziale Medien transnational Solidarität, Empörung und Mobilisierung zu konkreten Protestaktionen stattfinden. $\mathrm{Ob}$ als transnationales Mitgefühl nach Terroranschlägen (\#JeSuisCharlie) oder als globale Empörung (\#HongkongProtests), Emotionen werden durch soziale Medien erzeugt, mobilisiert und zirkulieren in hoher zeitlicher Dynamik. Die niedrigschwelligen Artikulationsmöglichkeiten in sozialen Medien und damit verbundenen Ermächtigungsoptionen (\#aufschrei; \#MeToo) ermöglichen es, zuvor tabuisierte Themen öffentlich werden zu lassen, damit Grenzen zwischen Persönlichem und Öffentlichem zu verschieben und so Scham in Empörung, ,unschuldiges ‘ Nicht-Wissen in Komplizenschaft, Desinteresse in kollektive Verantwortung umzuwandeln.

Formen der Zeugenschaft, die früher exklusiv oder zuvörderst professionellen Beobachter*innen (zumeist Journalist*innen) vorbehalten waren, finden mithilfe mobiler Videotechnik und synchroner Übertragungsoptionen von Smartphones über digitale Netzwerke alltäglich und multiperspektivisch statt. Zuvor als ,objektive' Beobachterpositionen beschriebene Zeugnisse werden damit herausgefordert. Als „affective witnessing “ (Lehmann, Roth, und Schankweiler 2019) lässt sich dabei jene Form von Augenzeugenschaft beschreiben, bei der das affektive Verhältnis von Zeug*in und Bezeugtem im (Video-)Zeugnis sichtbar zu Tage tritt. Augenzeugenvideos öffentlicher Proteste (z. B. während des sogenannten ,Arabischen Frühlings` in Ägypten) wie auch Videodokumente (z. B. der Misshandlung von Gefangenen im US-amerikanischen Gefängnis von Abu Ghraib) machen den affektiven Gehalt dieser Video-Zeugnisse deutlich erkennbar. Smit et al. (2017) beobachten in der öffentlichen Wahrnehmung des Syrien-Krieges, dass diese Formen affektiver Zeugenschaft, die auf YouTube verfüg- 
bar sind, zunehmend journalistisch-distanzierte, beobachtende Positionierungen ersetzen. Als Charakteristika eines solchen affektiven Erinnerungsarchivs nennen sie dabei Unvorhersehbarkeit, Unberechenbarkeit und den Live-Charakter.

Nikunen $(2018 ; 2019)$ hat sich mit Formen von Protest und Engagement durch soziale Medien (social media activism) insbesondere im Umfeld der sogenannten europäischen Flüchtlingskrise beschäftigt. Sie identifiziert den strategischen Einsatz von Kommunikation über soziale Medien als affektive Mobilisierung sowohl bei nationalistischen Anti-Migrationsprotesten als auch bei Initiativen, die Geflüchtete im Ankunftsland unterstützen. Als „,feel-good humanitarianism“ beschreibt sie dabei kritisch jene Formen der Unterstützung und des Mitgefühls, die weniger an struktureller politischer Veränderung, denn am subjektiven Wohlbefinden der Unterstützer*innen interessiert sind. Hier wird sichtbar, dass die soziale Entbettung durch digitale Medien das stolze Präsentieren des eigenen Mitgefühls gegenüber einem möglichst großen Kreis von Betrachter*innen anstelle direkter Interaktion mit Betroffenen - Teil eines komplexen Emotionsmanagements ist. Nicht die Adressierung eines unmittelbaren Gegenübers, sondern die Affizierung eines körperlich abwesenden Kreises von Users/Followers sind Ziel und Effekt dieser Formen von social media activism. Vor diesem Hintergrund werden Fragen der Nachhaltigkeit solcher Proteste und Mobilisierungen kontrovers diskutiert. Aber Proteste beschränken sich nicht allein auf clicktivism, sondern treten oftmals als Verschränkung von On- und Offline Aktionen auf, wie die Proteste nach dem Amtsantritt des amerikanischen Präsidenten (Women's March) in Washington und weltweit deutlich gezeigt haben. Insbesondere in der feministischen Medienforschung sind Formen des hashtag activism als transnationale Protestformen eingehend untersucht worden (vgl. als Überblick Lünenborg und Raetzsch 2018, S. 25-28). Ob in der Wiederaneignung visueller Repräsentationen und Körperbilder (\#FeministSelfie), der Rückeroberung des öffentlichen Raums (Hollaback! eine geodaten- und fotobasierte Form des Protests gegen Alltagssexismus) oder der offensiven Herstellung eines ,öffentlichen Selbst' als politischer Ermächtigung junger Frauen (Keller 2012) - durch affektive Medienpraktiken können Interventionen in öffentliche Diskurse initiiert, Sichtbarkeit erzeugt und damit Handlungsmacht (wieder)hergestellt werden.

Den Einsatz von Memes als spezifische Form digitaler Diskursintervention diskutieren Stehling et al. (2018) am Beispiel von \#distractinglysexy. Memes lassen sich als eigenständige symbolische Form affektiver Medienpraktik in sozialen Medien begreifen, mittels derer um Deutungshoheit zu kontroversen Themen gerungen wird. Shifman fasst dieses Ringen als semiotischen Aushandlungsprozess: „At any given 
moment, many memes are competing for the attention of hosts. However, only memes suited to their socio-cultural environment will spread successfully; the others will become extinct. Memes can be ideas, symbols or practices formed in diverse incarnations, such as melodies, catch-phrases, clothing fashion or architectural styles. While some memes are global, others are more culture specific, shaping collective actions and mindsets" (Shifman 2011, S. 188-189). Als Protest gegen sexistische Abwertungen durch den Nobelpreisträger Tim Hunt im Sommer 2015 posten Wissenschaftler*innen weltweit Bilder und Texte, die sie in ihrem Arbeitsumfeld sichtbar machen. So entsteht eine transnationale Gemeinschaft von Wissenschaftler*innen, die sich selbst und ihre Körper visuell inszenieren (Stehling, Brantner und Lobinger 2018, S. 229-236). Durch die multimodalen digitalen Elemente von Memes, die von zahlreichen Akteur*innen kreativ erzeugt bzw. modifiziert und in der Zirkulation von Benutzer*innen geteilt werden, erfährt das Ausgangselement (Foto oder Video) durch Text oder Graphik fortlaufend Ergänzung, Ironisierung und Re-Kontextualisierung. Der kreative Prozess der Gestaltung und Aneignung visuell-textueller Repräsentationen von Wissenschaft erzeugt Geschmacksgemeinschaften, die miteinander das Vergnügen der Umdeutung, Ironisierung und Persiflage teilen. Anstelle sich als Opfer von Diskriminierung zu präsentieren, wenden die Beteiligten ihr Tun in die subversive Aneignung der eigenen Rolle und repräsentieren sich als Verkörperung von Wissenschaft jenseits stereotypisierender Geschlechterrollenzuschreibungen - aus Ohnmacht und Wut wird so subversive Provokation und heitere Souveränität.

\section{Affective publics - Zur Relevanz von Emotionen für die Konstituierung von Öffentlichkeiten}

Die Vielzahl beschriebener affektiver Medienpraktiken bleibt nicht allein auf den Umgang mit sozialen Medien beschränkt. Sie gestalten weiterreichende Dispositionen im Umgang mit (digitalen) Medien, bei denen die Zuwendung zu Medieninhalten, die Interaktion mit Medientechnologien sowie die Adressierung als Nutzer*in nachhaltig affektiv strukturiert wird. Tradierte Konzepte zur Konstituierung von Öffentlichkeit, die primär einem rationalen Diskurs verpflichtet sind (Habermas 1962), können diese affektiv grundierten Dynamiken nicht angemessen erfassen und erklären. Als ,affective news stream" beschreibt Papacharissi die hybriden Medienerzählungen, in denen sich professionell aufbereitete Nachrichten mit user generated content aus sozialen Medien verbinden: „In telling their own stories and engaging in meaningmaking practices, storytelling audiences deconstruct and reconstruct storytelling conventions [...]. The liminality inherent in these practices permits citizens to make space for their own place in 
the story, and potentially lay claim to how these stories combine to form histories." (Papacharissi 2014, S. 28) In der Analyse der Social-Media-Berichterstattung über den Arabischen Frühling im Jahr 2011 (\#egypt) zeichnet sie den hybriden Charakter des Storytelling auf Twitter nach, bei dem Fakten, Bewertungen, persönliche Beschreibungen und geteilte Gefühle unauflöslich miteinander verbunden sind. Als affektive Dynamik erfasst sie dabei ,emotive gestures that blend opinion expression, phatic communication, and emotion into one, not unlike many of our utterances in everyday life“" (Papacharissi 2015, S. 95; Papacharissi und de Fatima Oliveira 2012).

In diesem Zusammenwirken unterschiedlicher Kommunikationselemente, diverser kommunikativer Modi und partizipativer Optionen durch soziale Medien entstehen Formen von Öffentlichkeit, für die Affekte konstitutiv sind. Als affective public beschreibt Papacharissi "networked public formations that are mobilized and connected or disconnected through expressions of sentiment" (2015, S. 125). Während Papacharissi eine trennscharfe und dichotome Unterscheidung zwischen Affekt und Emotion zugrunde legt und das Affektive, dem Philosophen Massumi (2002) folgend, kategorisch als nicht-diskursiv und bedeutungstragend begreift (vgl. dazu oben der Abschnitt Emotionen und Affekte als Elemente sozialer Ordnung), erscheint ein solch eingeschränktes Verständnis des Affektiven nicht notwendig und überzeugend (vgl. auch Nikunen 2019). Als affective publics soll hier vielmehr das performative Hervorbringen von Formen des Öffentlichen durch das relationale Gefüge von Medientechnologien, verkörperten Praktiken der Nutzer*innen und multimodalen Medientexten verstanden werden. Affekte werden dabei als zentral für das Hervorbringen von Öffentlichkeiten (im Plural) begriffen. Der analytische Blick auf das Affektive ermöglicht es, den relationalen, performativen und prozessualen Charakter des Öffentlichwerdens zu erfassen (Lünenborg 2019, S. 319). Um der Bedeutung nutzergenerierter Inhalte und der dynamischen Interaktion in den Strukturen digitaler Plattformen gerecht zu werden, erscheint es erforderlich, genau jene dort wirksam werdenden affektiven Dynamiken in den Fokus zu rücken. So werden affektive Kräfte nicht als Gegenpol zum rationalen Diskurs, sondern stets mit dem Diskursiven verschränkt als basales Element sozialen Handelns sichtbar. Mit der ubiquitären Verbreitung digitaler Netzwerkmedien sind professionell erzeugte Medienangebote in ihrem Ringen um Sichtbarkeit und Deutungshoheit stets den affektiven Dynamiken sozialer Medien ausgesetzt. Sie können dabei als Verstärkung, Kontrastierung oder Irritation Wirkung entfalten.

Die technologische Infrastruktur prägt und strukturiert diese Formierungen des Öffentlichen, hergestellt jedoch werden sie in den affektiven Praktiken der Nutzer*innen. Als 
hochgradig dynamische, zeitlich gebundene und oftmals nur flüchtige Erscheinungen lässt sich Öffentliches in diesem Sinne als fluide Struktur begreifen. Phänomene wie Ansteckung, Mimikry oder Viralität - ohne damit zu simple Gleichsetzungen mit biologischen Vorgängen assoziieren zu wollen - werden mit Konzepten des Affekts, der auch nicht-intentionales und un-/vorbewusstes Handeln einschließt, beschreibbar und erklärbar.

Eine solche Diagnose zeitgenössischer Öffentlichkeiten als affektive Öffentlichkeiten reiht sich ein in weitergehende Gesellschaftsanalysen als affective capitalism. $\mathrm{Ob}$ auf der Ebene betrieblicher Strukturen oder nationalstaatlicher Zugehörigkeiten, affective capitalism erlaubt Formen des affektiven Regierens jenseits von ökonomischer Belohnung oder gesetzlicher Regulation (Hardt 1999; Hardt und Negri 2000). Hier zeigt sich, in welchem Maße im zeitgenössischen post-industriellen Wirtschaftssystem Affekte gezielt verfügbar und nutzbar gemacht werden (Penz und Sauer 2016). Nicht allein körperliche und geistige Arbeitskraft werden als verfügbar betrachtet, sondern der zunehmend intensivierte Gebrauch von körperlich-affektiven Potenzialen, die vollständige Zurichtung von Interessen, Bedürfnissen, Lust und Unlust entlang kapitalistischer Verwertbarkeit kennzeichnet diese Formen affektiven Kapitals (Gregg 2011). Dazu gehört etwa die Bereitschaft, als Kund*in fortlaufend unentgeltlich an Produktoptimierungen beteiligt zu sein, die hochmotivierte grenzenlose Verfügbarkeit von Arbeitskraft in digitalen Start-ups ebenso wie die freiwillige Inkorporierung metrischer Logiken in Freizeit und Arbeitszeit, die subjektiv möglicherweise als individuelle Freiheiten erlebt werden.

\section{Fazit}

Werden Emotionen als Bausteine sozialer Ordnung verstanden, so wird erkennbar, dass die Architektur sozialer Medien, die in hohem Maße auf Intensivierung und Regulation von Emotionen abzielt, an der Re-Strukturierung eben dieser sozialen Ordnung mitbeteiligt ist. So lassen sich beschleunigte affektive Dynamiken in öffentlichen Debatten auch auf die spezifischen Affordanzen digitaler Plattformen und deren ökonomische Triebkräfte zurückführen. Jedoch erscheint es zu simpel, hier von einem einseitig gerichteten Ursache-Wirkung-Zusammenhang auszugehen, wie es der GrünenVorsitzende Robert Habeck gemacht hat, wenn er den Kurznachrichtendienst Twitter als Stimulus aggressiven Kommunikationsverhaltens beschreibt. Statt solcher Kausalitätszuschreibung erscheint es angemessener, ein wechselseitig konstituierendes Verhältnis von Emotionen und digitalen Netzwerkmedien zu Grunde zu legen. Einerseits 
erzeugen soziale Medien spezifische emotionale Artikulationen und Praktiken. Zugleich jedoch werden soziale Medien erst in ihrer spezifischen Nutzung und Aneignung wirksam. Die Gestaltung von Memes oder die Nutzung von Emoticons sind dabei Beispiele dafür, wie Nutzer*innen eigenständige, zuweilen subversive Ausdrucksweisen entwickeln, bei denen der Erzeugung und Kommunikation von Emotionen eine hervorgehobene Bedeutung zukommt. Doch auch jenseits solch semiotischer Mittel bieten soziale Medien in besonderer Weise Gelegenheit, Emotionsgemeinschaften (Wahl-Jorgensen 2019) zu bilden.

Unterhalb der Ebene elaborierter, diskreter Emotionen steht mit dem Begriff des Affekts ein Konzept zur Verfügung, das die relationale Verbindung von menschlichen Körpern und Medientechnologie in den Blick nimmt. Die ubiquitäre Verbreitung mobiler vernetzter Kommunikationstechnologien stellt die Voraussetzung dafür dar, dass über soziale Medien affektive Medienpraktiken dynamisch und vielfältig ihren Ausdruck finden. Es sind diese Formen der Affizierung, die Voraussetzung für Partizipation und Teilhabe an Öffentlichkeiten sind. Zugleich jedoch können solche Affizierungen auch zum Treiber von Exklusion werden, verstanden als affektiv basierte Markierung von Differenz und Ungleichheit. Mit dem Konzept der affective publics wird genau dieser Dynamik öffentlicher Artikulationen Rechnung getragen. Die Formierung von Öffentlichkeiten in digitalen vernetzten Kommunikationsräumen lässt sich - so die grundlegende Annahme - nicht allein beschränkt auf den rationalen Austausch von Argumenten verstehen. Vielmehr muss dafür genau jenes interaktive Kommunikationsgeflecht in den Blick genommen werden, das Informationen ebenso wie Stimmungen, Gefühle und Bewertungen einschließt. Denn eben dadurch werden Menschen informiert, mobilisiert und beteiligt. Mit genau diesen Formen jedoch können sie gleichermaßen beschimpft, beleidigt und ausgeschlossen werden.

\section{Literatur}

Ahmed, S. 2004a. Affective Economies. Social Text 22 (2): 117-139.

Ahmed, Sara. 2004b. The Cultural Politics of Emotion. Edinburgh: Edinburgh University Press.

Anderson, C. W. 2018. Apostles of Certainty: Data Journalism and the Politics of Doubt. New York, NY: Oxford University Press.

Banks, J. 2010. Regulating Hate Speech Online. International Review of Law, Computers \& Technology 24 (3): 233-39.

Blackman, Lisa. 2012. Immaterial Bodies: Affect, Embodiment, Mediation. London/New York, NY: SAGE.

Blackman, L., und C. Venn. 2010. Affect. Body \& Society 16 (1): 7-28. 
Boler, M., und E. Davis. 2018. The Affective Politics of the "Post-Truth" Era: Feeling Rules and Networked Subjectivity. Emotion, Space and Society 27: 75-85.

Clough, Patricia Ticineto, und Jean Halley. 2007. The Affective Turn: Theorizing the Social. Durham, NC/London: Duke University Press.

Dijck, José van. 2013. The Culture of Connectivity. A Critical History of Social Media. New York, NY: Oxford University Press.

Ekman, P. 1992. An Argument for Basic Emotions. Cognition and Emotion 6 (3-4): 169-200.

Erjavec, K., und M. Poler Kovačič. 2012. "You Don’t Understand, This Is a New War!” Analysis of Hate Speech in News Web Sites' Comments. Mass Communication and Society 15 (6): 899920.

Faris, Robert, Amar Ashar, Urs Gasser und Daisy Joo. 2016. Understanding Harmful Speech Online. Cambridge, MA: Berkman Klein Center for Internet \& Society at Harvard University. https://dash.harvard.edu/handle/1/38022941. Zugegriffen: 26.02.2020.

Frevert, Ute. 2017. Die Politik der Demütigung: Schauplätze von Macht und Ohnmacht. Frankfurt am Main: S. Fischer.

Gagliardone, I. 2019. Defining Online Hate and Its ,Public Lives': What is the Place for ,Extreme Speech“? International Journal of Communication 13: 3068-3087.

Gregg, Melissa. 2011. Work's Intimacy. Cambridge, UK/Malden, MA: Polity Press.

Gregg, Melissa, und Gregory J. Seigworth. 2010. The Affect Theory Reader. Durham, NC: Duke University Press.

Haarkötter, Hektor. 2016. Empörungskaskaden und rhetorische Strategien in Shitstorms. Eine empirische Analyse des User-Verhaltens in ausgewählten Facebook-Shitstorms. In Shitstorms und andere Nettigkeiten. Über die Grenzen der Kommunikation in Social Media, Hrsg. Hektor Haarkötter, 17-50. Baden-Baden: Nomos.

Habeck, Robert. 2019. Bye bye, twitter und Facebook. Robert Habeck (Blog). Eintrag vom 7. Januar 2019. https://www.robert-habeck.de/texte/blog/bye-bye-twitter-und-facebook/. Zugegriffen: 26.02 .2020 .

Habermas, Jürgen. 1962. Strukturwandel der Öffentlichkeit. Untersuchungen zu einer Kategorie der bürgerlichen Gesellschaft. Frankfurt am Main: Suhrkamp.

Hardt, M. 1999. Affective Labor. boundary 2 - an international journal of literature and culture 26 (2): 89-100.

Hardt, Michael, und Antonio Negri. 2000. Empire. Cambridge, MA: Harvard University Press.

Hochschild, A. R. 1979. Emotion Work, Feeling Rules, and Social Structure. American Journal of Sociology 85 (3): 551-575.

Illouz, Eva. 2003. Der Konsum der Romantik. Liebe und die kulturellen Widersprüche des Kapitalismus. Frankfurt am Main/New York, NY: Campus.

Illouz, Eva. 2016. Gefühle in Zeiten des Kapitalismus. Frankfurt am Main: Suhrkamp Verlag. 
Illouz, Eva. 2018. Einleitung: Gefühle als Waren. In Wa(h)re Gefühle. Authentizität im Konsumkapitalismus, Hrsg. Eva Illouz, 13-48. Berlin: Suhrkamp.

Keller, J. M. 2012. Virtual Feminisms: Girls' blogging communities, feminist activism, and participatory politics. Information, Communication \& Society 15 (3): 429-447.

Konijn, Elly A. 2013. The role of emotion in media use and effects. In The Oxford Handbook of Media Psychology, Hrsg. Karen E. Dill, 187-248. New York, NY: Oxford University Press.

Koppetsch, Cornelia. 2019. Die Gesellschaft des Zorns: Rechtspopulismus im globalen Zeitalter. Bielefeld: transcript.

Kramp, Leif, und Stephan Weichert. 2018. Hasskommentare im Netz. Steuerungsstrategien für Redaktionen, Hrsg. Landesanstalt für Medien NRW, Düsseldorf. Leipzig: VISTAS Verlag.

Lehmann, Hauke, Hans Roth, und Kerstin Schankweiler. 2019. Affective economy. In Affective Societies - Key Concepts, Hrsg. Jan Slaby und Christian von Scheve, 140-151. London: Routldege.

Ley, Hannes. 2018. \#ichbinhier: zusammen gegen Fake News und Hass im Netz. Köln: DuMont.

Lünenborg, Margreth. 2019. Affective Publics. In Affective Societies - Key Concepts, Hrsg. Jan Slaby und Christian von Scheve, 319-329. Routledge: London.

Lünenborg, M. und T. Maier. 2018. The Turn to Affect and Emotion in Media Studies. Media and Communication 6 (3): 1-4.

Lünenborg, Margreth. 2019. Analyzing Affective Media Practices by the Use of Video Analysis. In Analyzing Affective Societies: Methods and Methodologies, Hrsg. Antje Kahl, 140-161. New York: Routledge.

Lünenborg, Margreth, und Christoph Raetzsch. 2018. From Public Sphere to Performative Publics: Developing Media Practice as an Analytic Model. In Media Practices, Social Movements, and Performativity: Transdisciplinary Approaches, Hrsg. Margreth Lünenborg, Christoph Raetzsch und Susanne Foellmer, 13-35. New York: Routledge.

Malinowska, A. und T. Miller. 2017. Sensitive Media. Open Cultural Studies 1 (1): 660-665.

Massumi, Brian. 2002. Parables for the Virtual: Movement, Affect, Sensation. Durham, NC: Duke University Press.

Naab, T. K., A. Kalch und T. G. K. Meitz. 2018. Flagging Uncivil User Comments: Effects of Intervention Information, Type of Victim, and Response Comments on Bystander Behavior. New Media \& Society 20 (2): 777-795.

Neckel, Sighard. 1991. Status und Scham. Zur symbolischen Reproduktion sozialer Ungleichheit. Frankfurt am Main/New York, NY: Campus Verlag.

Neue deutsche Medienmacher*innen. 2018. No Hate Speech Movement. Neue deutsche Medienmacher*innen gegen Hassrede im Netz. https://www.neuemedienmacher.de/projekte/no-hatespeech-movement/. Zugegriffen: 26.02.2020.

Nikunen, K. 2018. From Irony to Solidarity: Affective Practice and Social Media Activism. Studies of Transition States and Societies 10 (2): 10-21. 
Nikunen, Kaarina. 2019. Media Solidarities. Emotions, Power and Justice in the Digital Age. London: Sage.

Papacharissi, Z. 2014. Toward New Journalism(s): Affective news, hybridity, and liminal spaces. Journalism Studies 16 (1): 27-40.

Papacharissi, Zizi. 2015. Affective Publics: Sentiment, Technology, and Politics. New York, NY: Oxford University Press.

Papacharissi, Z. und M. de Fatima Oliveira. 2012. Affective News and Networked Publics: The Rhythms of News Storytelling on \#Egypt. Journal of Communication 62 (2): 266-282.

Penz, Otto, und Birgit Sauer. 2016. Affektives Kapital. Die Ökonomisierung der Gefühle im Arbeitsleben. Frankfurt/New York, NY: Campus Verlag.

Pfeffer, J., T. Zorbach und K. M. Carley. 2014. Understanding Online Firestorms: Negative Wordof-Mouth Dynamics in Social Media Networks. Journal of Marketing Communications 20 (12): 117-128.

Reckwitz, Andreas. 2017. Practices and their affects. In The Nexus of Practices: Connections, constellations, practitioners, Hrsg. Allison Hui, Theodore Schatzki und Elizabeth Shove, 114-125. London: Routledge.

Sauer, B. und O. Penz. 2014. Affektive Subjektivierung: Arbeit und Geschlecht. Freiburger Zeitschrift für GeschlechterStudien FZG 20 (2): 79-94.

Scherer, K. R. 2005. What are emotions? And how can they be measured? Social Science Information 44 (4): 695-729.

Scheve, Christian von und Anna Lea Berg. 2018. Affekt als analytische Kategorie der Sozialforschung. In Stimmungen und Atmosphären. Zur Affektivität des Sozialen, Hrsg. Larissa Pfaller und Basil Wiesse, 27-51. Wiesbaden: Springer VS.

Sellars, Andrew F. 2016. Defining Hate Speech. Berkman Klein Center for Internet \& Society Research Publication, Nr. 2016-20. Boston University School of Law, Public Law Research Paper No. 16-48. https://papers.ssrn.com/sol3/papers.cfm?abstract_id=2882244. Zugegriffen: 26.02.2020.

Shifman, L. 2011. An Anatomy of a YouTube Meme. New Media \& Society 14 (2): 187-203.

Slaby, Jan. 2018. Drei Haltungen der Affect Studies. In Stimmungen und Atmosphären: Zur Affektivität des Sozialen, Hrsg. Larissa Pfaller und Basil Wiesse, 53-81. Wiesbaden: Springer VS.

Smit, R., A. Heinrich und M. Broersma. 2017. Witnessing in the New Memory Ecology: Memory Construction of the Syrian Conflict on YouTube. New Media \& Society 19 (2): 289-307.

Spiller, Ralf, und Thomas Hintzen. 2016. Empörungswellen im Internet: Genese, Gegenstrategien und Auswirkungen. In Shitstorms und andere Nettigkeiten. Über die Grenzen der Kommunikation in Social Media, Hrsg. Hektor Haarkötter, 109-122. Baden-Baden: Nomos.

Stehling, Miriam, Cornelia Brantner und Katharina Lobinger. 2018. Meme als Diskursintervention: Körperbilder gegen Sexismus am Beispiel von \#distractinglysexy. In Körperbilder - Körperpraktiken. Visualisierung und Vergeschlechtlichung von Körpern in Medienkulturen, Hrsg. 
Elke Grittmann, Katharina Lobinger, Irene Neverla, und Monika Pater, 225-244. Köln: Herbert von Halem Verlag.

Wagner, Elke. 2019. Intimisierte Öffentlichkeiten: Pöbeleien, Shitstorms und Emotionen auf Facebook. Bielefeld: transcript.

Wahl-Jorgensen, Karin. 2018. Emotions, Media and Politics. Hoboken, NJ: Wiley.

Wahl-Jorgensen, Karin. 2019. Creating an Emotional Community: The Negotiation of Anger and Resistance to Donald Trump. In Media and the Politics of Offence, Hrsg. Anne Graefer, 47-63. Cham, CH:Palgrave Macmillan.

Wetherell, Margaret. 2012. Affect and Emotion: A New Social Science Understanding. Los Angeles/London: SAGE.

Ziegele, Marc, und Pablo B. Jost. 2016. Not Funny? The Effects of Factual Versus Sarcastic Journalistic Responses to Uncivil User Comments. Communication Research. doi: $10.1177 / 0093650216671854$.

Ziegele, Marc, Pablo Jost, Dennis Frieß und Teresa Naab. 2019. Aufräumen im Trollhaus. Zum Einfluss von Community-Managern und Aktionsgruppen in Kommentarspalten. DIID -Düsseldorf Institute for Internet and Democracy - Précis, April 2019: 1-12. https://diid.hhu.de/diidprecis/. Zugegriffen: 26.02.2020.

Ziegele, M., C. Koehler und M. Weber. 2018. Socially Destructive? Effects of Negative and Hateful User Comments on Readers' Donation Behavior toward Refugees and Homeless Persons. Journal of Broadcasting \& Electronic Media 62 (4): 636-653. 